\title{
Review of: "Lower probability and shorter duration of infections after COVID-19 vaccine correlate with anti- SARS-CoV-2 circulating IgGs"
}

\author{
K. Hari Prasad ${ }^{1}$
}

1 Apollo Hospitals

Potential competing interests: The author(s) declared that no potential competing interests exist.

Please find my observations as follows:

1. saliva can be successfully employed for SARS-CoV-2 detection by molecular assays with similar or higher sensitivity compared to the same assays applied on nasopharyngeal swabs,

2. Their data show that individuals who responded to vaccination based on the detection of anti-RBD antibodies were still susceptible to SARS-CoV-2 productive infection, suggesting caution, especially for healthcare workers that are daily in contact with fragile patients,

3. However, probability of infection after vaccination is rare and significantly less frequent compared to reinfection after natural infection, in particular in responders, which are the vast majority.

4. Furthermore, duration of infection in vaccinated individuals is significantly shorter to the ones observed post-natural infection, suggesting that post-vaccination viral shedding is likely very limited, recommending for a revision of the isolation policies, that could drastically reduce the time of quarantine, with clear important social and economic implications, and implications at the workplace. 